\title{
Oral Involvement in a Case of Chronic Graft Versus Host Disease
}

\author{
Apostolos Matiakis, ${ }^{1}$ Alexandros Kolokotronis, ${ }^{2}$ Demetrios Antoniades, ${ }^{2}$ and Anthi Asimaki ${ }^{3}$ \\ ${ }^{1}$ Private Practice, Thessaloniki, Greece \\ ${ }^{2}$ Department of Oral Medicine/Pathology, Dental School, Aristotle University of Thessaloniki, Greece \\ ${ }^{3}$ Department of Histopathology, G. Papanikolaou General Hospital, Thessaloniki, Greece
}

Correspondence to :

Alexandros Kolokotronis

E-mail : kdeod@cieel.gr

Keywords :

graft-versus-host disease, oral manifestations, oral ulcerations, licheinoid lesions, xerostomia

\begin{abstract}
This article describes a case of oral involvement in chronic graftversus-host disease (cGVHD) after hematopoietic cell transplantation. The patient suffered from painful oral ulcerations, especially on the tongue, lichenoid buccal mucosa lesions, and xerostomia. She also developed purpuric exanthema and scleroderma-like signs on her hands. Her history, in combination with clinical examination and histological findings of both lichenoid lesions and labial salivary glands, revealed cGVHD.
\end{abstract}

\section{Introduction}

Graft-versus-host disease (GVHD) can occur in recipients of allogeneic bone marrow when immunocompetent graft cells ( $\mathrm{T}$ cells) react against an immunodeficient host. Bone marrow transplantation (B.M.T.) is used as a therapeutic approach to blood malignancies, severe aplastic anemia, and some metabolic disorders $(1,2)$.

On 1966 Billingham described three criteria necessary for the development of GVHD: 1) the graft must contain immunologically competent cells, 2) the host must appear foreign to the graft, and 3) the host must be incapable of reacting against the graft (3).

There are two types of GVHD. They differ in the time of development and the clinical manifestations. Acute GVHD (aGVHD) occurs within 100 days after B.M.T. and is characterized by dermatitis (skin rash), enteritis (diarrhea), hepatitis (liver dysfunction), and increased susceptibility to infections. Chronic GVHD (cGVHD) occurs after 100 days of B.M.T. and develops as a sequence of aGVHD or occurs de novo in recipients who never showed aGVHD. cGVHD involves the oral cavity, skin, gastrointestinal tract, lungs and liver. In the oral cavity, involvement ranges upwards of $80 \%$, including painful oral ulcerations, lichenoid lesions, atrophy of the buccal and labial mucosa, and dryness similar to that of Sjögren's syndrome. The skin can exhibit rash, lichen planuslike papules, sclerodermatous changes with necrosis and ulcers on acral and pressure sites, hair loss. In the gastrointestinal tract, cGVHD especially affects the esophagus with dysphagia, and insidious weight loss can occur. Bronchiolitis leads to obstructive lung disease, and the patient can experience portal hypertension, cirrhosis, and death from hepatic failure. Other findings include vaginitis, autoimmune thrombocytopenia, and anemia (3-6).

\section{Case Report}

A 30-year-old woman was referred from the hematological clinic G. Papanicolaou Hospital, to department of Oral Medicine and Pathology Dental School University of Thessaloniki, because she had severe oral pain, buccal mucosal lesions, and xerostomia. The patient developed severe painful ulcerations on the right buccal mucosa, palate mucosa, and on the tongue (Fig. 1). Her buccal mucosa was dry and, especially on the right side, had profound lichenoid lesions, and her hands had rash and sclerodermalike signs (Fig. 2). Her medical history included bone marrow transplantation (B.M.T.) because of chronic myelogenous leukemia, three months ago.

Biopsy of buccal mucosa (Fig. 3, 4). labial salivary gland (Fig. 5, 6). as well as skin (Fig. 7). was perfor- 
med, and the histopathological findings were GVHD compatible. The patient had already been checked for HSV, CMV, and EBV and was found negative. Mucosal scrapings stained with Periodic - Acid Schiff (PAS) were negative for Candida. Patient's history in combination with oral examination findings as well as histological findings revealed cGVHD.

The patient was already under prednizolone and

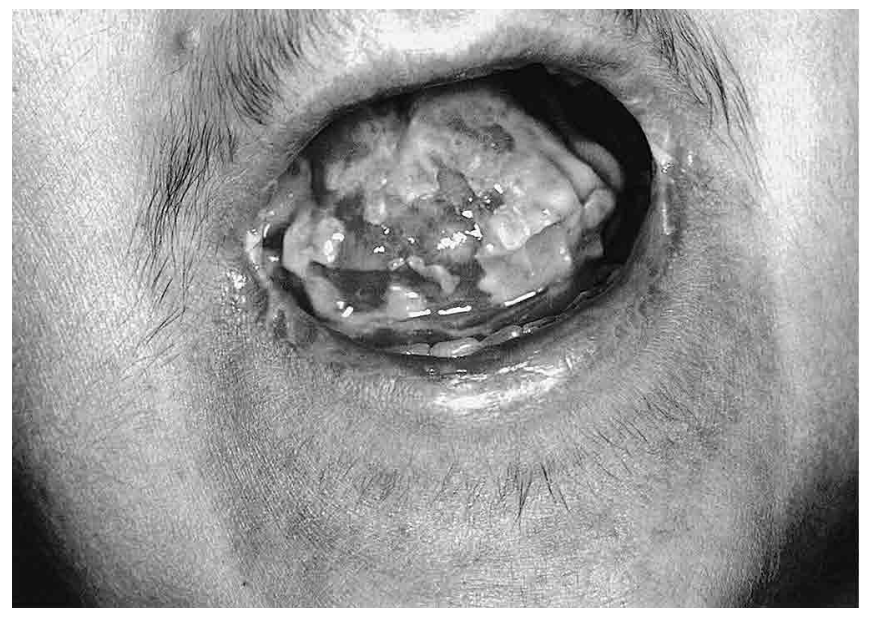

Fig. 1. Clinical view of the tongue, demonstrating the oral ulcerations.

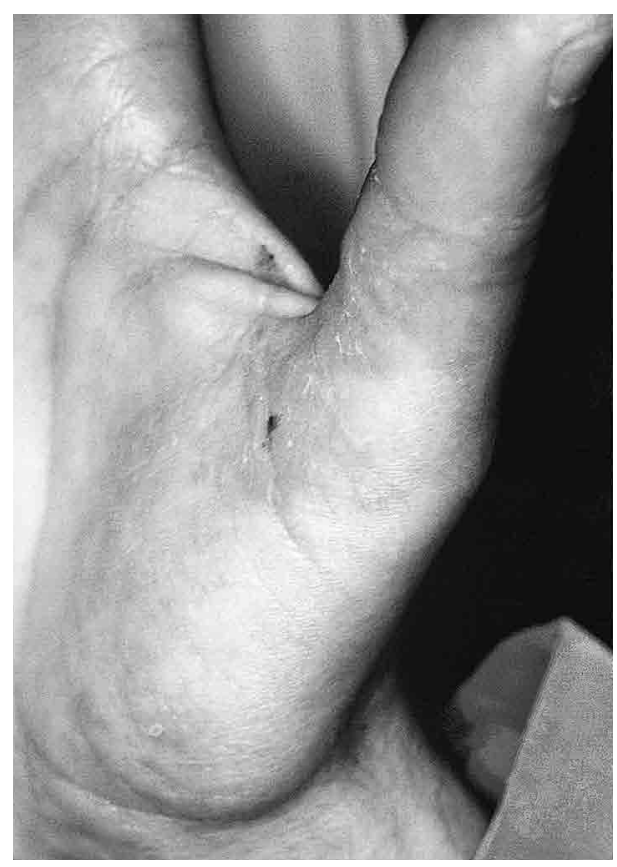

Fig. 2. Skin rash and sclerodermalike signs on patient's hands. cyclosporine treatment, so further treatment of the oral lesions was unnecessary.

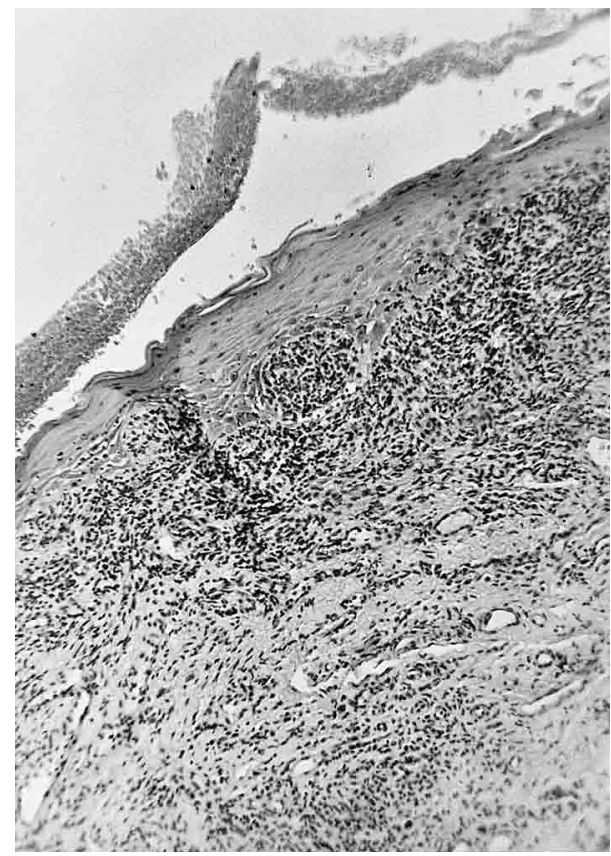

Fig. 3. A prominent lichenoid reaction in the oral mucosa. Image compatible with cGVHD (original manifestation $\mathrm{H}-\mathrm{E}$ $\times 200)$.

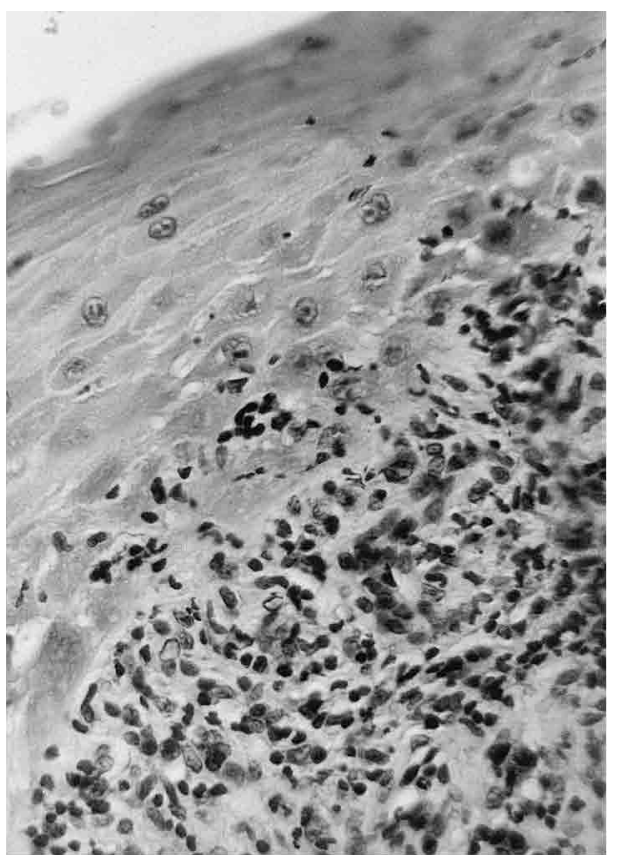

Fig. 4. Same as in Fig. 3 (original manifestation $\mathrm{H}-\mathrm{E} \times 400$ ). 


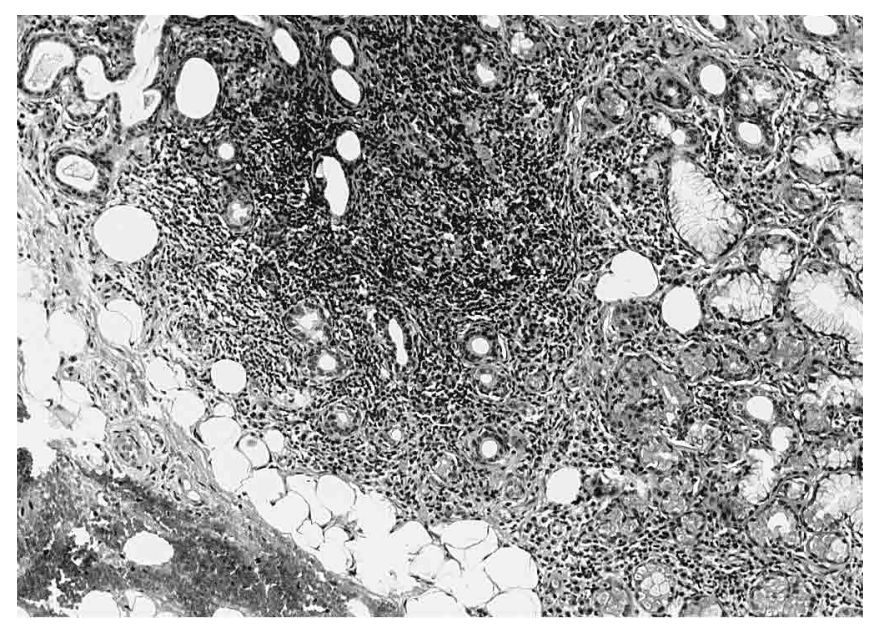

Fig. 5. Diffuse lymphocytic infiltration (original manifestation $\mathrm{H}^{-} \mathrm{E} \times 25$ ).

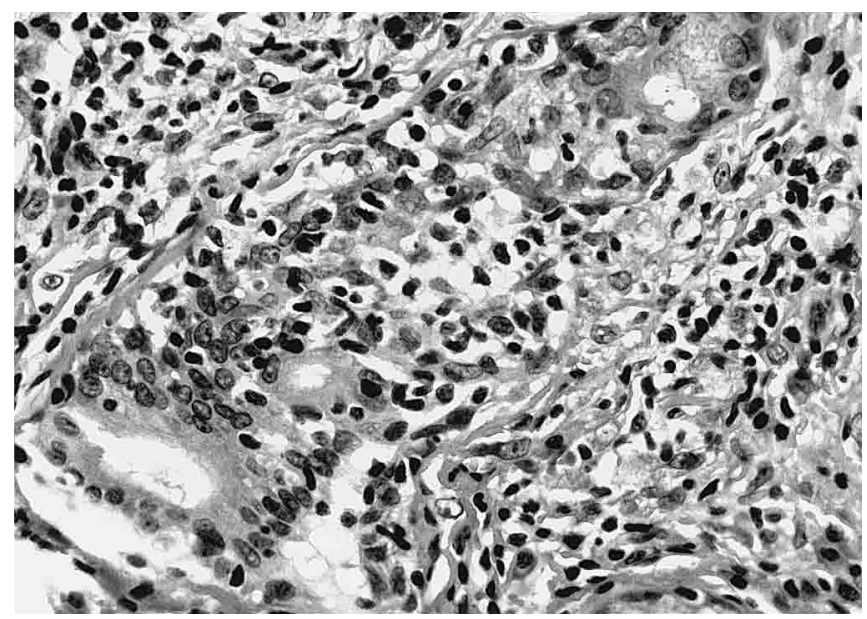

Fig. 6. Intraepithelial lymphocytic infiltration (original manifestation $\mathrm{H}-\mathrm{E} \times 200)$.

\section{Discussion}

Graft-versus-host disease is one of the most common problems that follow bone marrow transplantation, (1). Approximately $80 \%$ of cGVHD patients present oral manifestations including oral ulcerations, oral lichenoid lesions, atrophy of oral mucosa and xerostomia similar to that in Sjögren's syndrome $(1,3,4)$.

Ulcerations of various sizes may be throughout the oral cavity. Their etiology is of interest concerning their differential diagnosis. An immunoincompetent hematopoietic cell recipient may develop oral ulcerations as a result of bacterial, viral (especially HSV,

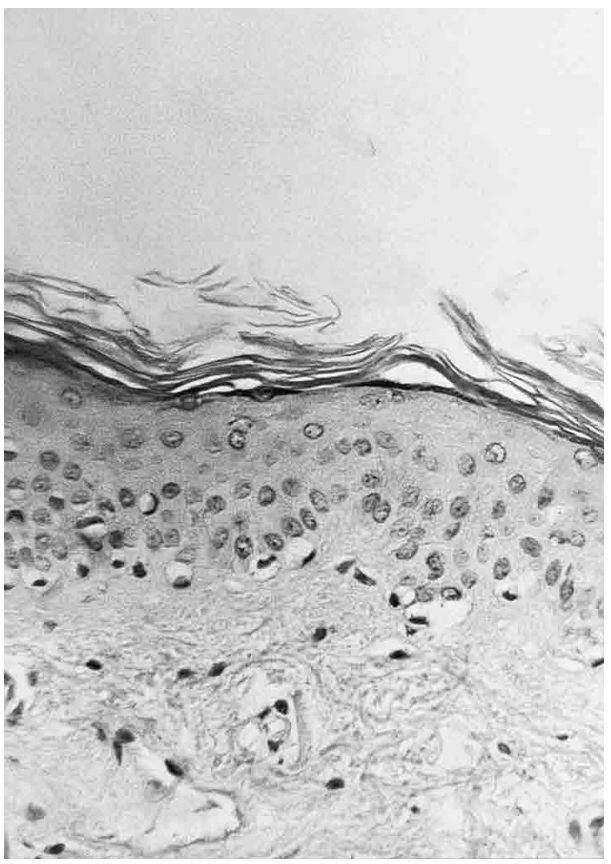

Fig. 7. Skin cGVHD. The epidermis is atrophic. The dermis shows thickened and hyalinized collagen bundles with destruction of adnexal structures (original manifestation $\mathrm{H}-\mathrm{E} \times 400)$

EBV, CMV) or fungal (Candida) infections (1). In order to prevent those infections, these patients often receive antibacterial, antiviral and antifungal prophylaxis $(1,2)$.

Also, oral ulcerations may develop as a side effect after chemotherapy, especially methotrexate (M.T. $\mathrm{X}$.) administration, which is an immunosuppressive agent that is administrated before allogeneic bone marrow transplantation or as a preventive treatment of GVHD (1, 3, 4). In addition, patients who have undergone total body irradiation in order to destroy the neoplasmatic haemopaietic cells will, most probably, have oral ulcerations as a side effect, as well as xerostomia, after the transplantation (1). The patient in this case report was negative for infections, and did not receive M.T.X. Also, she had not received total body irradiation.

The histological findings in buccal mucosa (BM)subepithelial lymphocytic infiltration and basal cell degeneration- as well as those in labial salivary glands (LSGs) help to diagnose and confirm the 
status of cGVHD (4).

Lichenoid lesions in oral mucosa may be localized on the buccal mucosa, tongue and labial mucosa and are among the main oral findings in cGVHD (6). Nevertheless, lichenoid lesions of cGVHD cannot be differentially diagnosed either clinically or histopathologically from oral lichen planus, which is not a rare disease (4).

Xerostomia is one of the main oral manifestations of cGVHD, and sometimes it is accompanied by xeropthalmia, which is why this type of cGVHD was called Sjögrenlike syndrome (7). Latest research, however, shows that there are characteristic histopathologic differences in labial salivary glands between cGVHD and Sjögren's syndrome. Especially, periductal lymphocytic infiltration is typical in Sjögren's syndrome but not in cGVHD patients, whose typical histological findings diffuse lymphocytic infiltration (4).

Dermal manifestations clinically resemble systematic erythematous lupus, lichen, Stevens- Johnson syndrome or scleroderma, as in the presented case, followed by histopathological findings not adequate for cGVHD diagnosis. These findings are important for the final diagnosis $(2,3)$.

The development of secondary malignant diseases after BMT stands among the complications in BMT patients $(8,9)$. Lymphohematopoietic malignancies are most frequent while solid malignant tumors as squamous cell carcinoma, melanoma, glioblastoma, and sarcoma are less frequent. Abdelsayed et al. report two young patients who suffered from GVHD after HCT. Those patients developed oral squamous cell carcinoma at anatomic sites that were affected from GVHD. The same authors, in a literature review, report eight more cases of oral cancer in GVHD patients (10).

The best treatment of GVHD is its prevention. Treatment with cyclosporine, along with M.T.X. and prednizolone, seems to have the best results (5). Certain authors suggest that $\mathrm{T}$-cell depletion of the transplanted marrow as a GVHD prophylaxis decreases oral mucosal lesions compared with a GVHD prophylaxis with a combination of cyclospor- ine and M.T.X. (11).

A thorough oral examination before BMT and through the period after the transplantation is of great importance for the prevention of oral and systematic infections because the oral cavity of the immunocompromised patient can be an entrance for microflora $(1,2)$.

\section{References}

1. Heimdahl A, Mattsson T, Dahllöf G, et al.: The oral cavity as a port of entry for early infections in patients treated with bone marrow transplantation. Oral Surg Oral Med Oral Pathol, 68 : 711-716, 1989.

2. Eggleston TI, Ziccardi VB, Lumerman H : Graft-versus-host disease. Case report and discussion. Oral Surg Oral Med Oral Pathol Oral Radiol Endod, 86 : 692-696, 1998.

3. Schubert MM and Sullivan KM: Recognition, incidence, and management of oral graft-versus-host disease. Vol. 9 : 135-143, 1990, NCI Monogr.

4. Nacamura S, Hiroki A, Shinohara M, et al.: Oral involvement in chronic graft-versus-host disease after allogeneic bone marrow transplantation. Oral Surg Oral Med Oral Pathol Oral Radiol Endod, 82 : 556-563, 1996.

5. Flowers ME, Kansu E, Sullivan KM : Pathophysiology and treatment of graft-versus-host disease. Hematol Oncol Clin North Am, 13 : 1091-1112, 1999.

6. Nikolatou-Galitis O, Kitra V, Van-Vliet-Constantinidou $\mathrm{C}$, et al.: The oral manifestations of chronic graft-versus-host disease (cGVHD) in paediatric allogeneic bone marrow transplant recipients. J Oral Pathol Med, 30 : 148-153, 2001.

7. Gratwohl AA, Moutsopoulos HM, Chused TM, et al. : Sjögren - type syndrome after allogeneic bone marrow transplantation. Ann Intern Med, 87: 703-706, 1977.

8. Whitherspoon RP, Fisher LD, Scotch G, Martin P, Sullivan KM, Sanders J, et al.: Secondary cancers after bone marrow transplantation for leukemia or aplastic anemia. N Eng J Med, 321: 784-9, 1989.

9. Deeg HJ, Socie G, Schoch G, Henry-Amar M, Whitherspoon RP, Devergie A, et al.: Malignancies after marrow transplantation for aplastic anemia and Fanconi anemia: a joint Seattle and Paris analysis of results in 700 patients. Blood, 87 : 386-92, 1996.

10. Abdelsayed RA, Sumner Ty, Allen CM, Treadway A, Ness GM, Penza SL: Oral precancerous and malignant lesions associated with graft-versus-host disease: Report of 2 cases. Oral Surg Oral Med Oral Pathol Oral Radiol Endod, 93 : 75-80, 2002. 
Int J Oral-Med Sci 3(2):105-109, 2004

11. Mattsson T, Heimdahl A, Dahllöf G, et al. : Oral and nutritional status in allogenetic marrow recipients treated with $\mathrm{t}^{-}$-cell depletion or cyclosporine combined with methotrexate to prevent graft- $^{-}$versus $^{-}$host-dis- $^{-}$ ease. Oral Surg Oral Med Oral Pathol, 74 : 34-40, 1992. 